\title{
INTNUM:
}

\section{Uma Ferramenta Computacional para Visualização e Cálculo dos Métodos de Integração Numérica}

\author{
Bruno Nunes Myrrha Ribeiro \\ Universidade Severino Sombra-USS, Vassouras-RJ - Centro \\ Universitário Geraldo Di Biase-UGB, Barra do Piraí-RJ \\ myrrhaugb@gmail.com
}

\author{
Carlos Vitor de Alencar Carvalho \\ Universidade Severino Sombra-USS, Vassouras-RJ \\ Centro Universitário de Volta Redonda-UniFOA, Volta Redonda-RJ - \\ Instituto Superior de Tecnologia - IST/FAETEC, Paracambi-RJ \\ cvitorc@gmail.com
}

\begin{abstract}
Resumo: O presente artigo destina-se a apresentação do software INTNUM. Trata-se de um software direcionado para o estudo do cálculo numérico e tem o objetivo de visualizar diferentes métodos de integração numérica, como: Retângulo, Trapézio e Simpson. Assim sendo, este software fornece o cálculo da área sob a função em uma variável, bem como possibilita a interação por meio do teclado, variando o número de partições e otimiza o valor numérico da integral. O sistema foi desenvolvido em linguagem de programação $\mathrm{C}++$, e utiliza o ambiente de desenvolvimento Microsoft Visual C++ 2008 Express Edition e sistema gráfico OpenGL. O uso do software tem como proposta complementar à aprendizagem na disciplina de Cálculo Numérico, além de proporcinar interatividade, visualização, $e$ otimização nos Métodos de Integração Numérica, contribuindo assim, no processo de ensino- aprendizagem.
\end{abstract}

Palavras-Chave: Software educativo. Cálculo numérico. Métodos de integração numérica. 


\title{
INTNUM:
}

\section{A Computational Tool for Visualization and Calculation Methods of Numerical Integration}

\begin{abstract}
This article is intended to INTNUM presentation software. This is a software directed to the study of numerical calculation and is intended to display different numerical integration methods, such as Rectangle, Trapezoid and Simpson. Thus, this software provides the calculation of the area under the function in one variable, and allows interaction via the keyboard, varying the number of partitions and optimize the numerical value of the integral.

The system was developed in programming language $C++$ using the development environment Microsoft Visual C++ 2008 Express Edition and OpenGL graphics system. The use of the software is proposed in order to complement the learning in the discipline of Numerical Calculus, providing interactivity, visualization, and optimization of numerical integration methods, thus helping in the teaching-learning process.
\end{abstract}

Keywords: educational software, numerical calculus, numerical integration methods.

\section{Introdução}

As grandes mudanças que aconteceram em relação à proposta pedagógica do século XXI estão relacionadas ao desenvolvimento das Tecnologias de Informação e Comunicação (TIC) e possibilitam novas práticas pedagógicas que favorecem o processo ensino-aprendizagem. Das práticas derivadas da informática aplicada à educação, o presente artigo direciona o desenvolvimento de software com enfoque educativo, precisamente classificado como, sistema de exercícios e práticas e simulação (Valente 1999).

No ensino da Engenharia existe uma grande demanda para aumentar a utilização de softwares educacionais em sala de aula. Algumas propostas podem ser vistas em Carvalho (2009), que apresenta um estudo da utilização do software OCTAVE para o apoio ao ensino do cálculo numérico. Carvalho (2005) desenvolveu um simulador para estudo do comportamento da ventilação cruzada em ambientes construídos.

Convém ressaltar que a aprendizagem deve ser feita pela informática e não da informática. Valendo-se dessa hipótese, a professora Regina Célia Haidt diz que:

(...) o emprego do computador no processo pedagógico, assim como o uso de qualquer tecnologia, exige do 
INTNUM: Uma Ferramenta Computacional para Visualização e Cálculo dos Métodos de Integração Numérica

Bruno Nunes Myrrha Ribeiro - Carlos Vitor de Alencar Carvalho

educador uma reflexão crítica. Refletir criticamente sobre o valor pedagógico da informática significa também refletir sobre as transformações da escola e repensar o futuro da educação (Haidt, 2001:215).

O espaço escolar é o lugar onde se constrói o conhecimento. Recursos que permitam e facilitem aos alunos a apropriação das experiências humanas consideradas como cultura, devem pertencer a este espaço como, segundo D’Ambrósio (p. 60, 2002):

“Como consequência na Educação. Não há como escapar. Ou os educadores adotam a teleinformática com absoluta normalidade, assim como o material impresso e a linguagem, ou serão atropelados no processo e inúteis na sua profissão. Procurem imaginar um professor que rejeita os meios mais tradicionais: falar, ver, ouvir, ler e escrever. Lamentavelmente ainda há alguns que só praticam o falar!”.

As considerações descritas acima foram às motivações para o desenvolvimento do software INTNUM. Este sistema foi desenvolvido em linguagem de programação C++, com técnicas de computação gráfica 2D (Foley et al. 1999), e com uso do sistema gráfico OpenGL, com objetivo pedagógico de auxiliar o processo ensino-aprendizagem na disciplina de Cálculo Numérico, sobretudo, os tópicos relativos aos métodos de integração numérica. Tratando-se da visualização, bem como do valor aproximado dos métodos Retângulo, Trapézio e Simpson.

O artigo apresenta-se da seguinte forma: na seção 2 são mostrados os desenvolvimentos matemáticos dos métodos da integração numérica e suas respectivas implementações; na 3 é apresentado o desenvolvimento do sistema INTNUM; na 4 são apresentados os softwares desenvolvidos e alguns exemplos da sua utilização como proposta complementar para apoio ao ensinoaprendizagem dos métodos de integração numérica.

\section{Métodos de Integração Numérica}

O cálculo de integrais numéricas aparece constantemente na resolução de diversos problemas que não podem ser resolvidos analiticamente, o que ocasiona aproximações com aplicação de alguns métodos, que na maioria dos casos não podem ser determinados explicitamente por fórmulas simples. Então, utiliza-se a resolução numérica pelas Somas de Riemann (Pereira 2008).

Dada uma função $\boldsymbol{f}(\boldsymbol{x})$ no intervalo $[\boldsymbol{a}, \boldsymbol{b}]$, dividido em $\boldsymbol{n}$ partições com comprimento $\boldsymbol{h}$, logo:

$$
h=\frac{b-a}{n}
$$


INTNUM: Uma Ferramenta Computacional para Visualização e Cálculo dos Métodos de Integração Numérica

Bruno Nunes Myrrha Ribeiro - Carlos Vitor de Alencar Carvalho

Sendo $\boldsymbol{x}_{\mathbf{0}}=\boldsymbol{a}$ e $\boldsymbol{x}_{\boldsymbol{n}}=\boldsymbol{b}$, a área limitada entre a curva, gerada pela função $\boldsymbol{f}(\boldsymbol{x})$, e o eixo das abscissas no intervalo é dada pela integral definida:

$$
\text { Area }=\int_{a}^{b} f(x) d x
$$

A dificuldade de integração em relação a algumas funções leva-nos à determinação de uma forma aproximada para o cálculo da área; que resulta no uso de métodos para o cálculo numérico de integrais.

\subsection{Método dos Retângulos}

Conhecido também como "Método do Ponto à Esquerda", consiste na aproximação numérica, por retângulos, em relação à área sob a função dada.

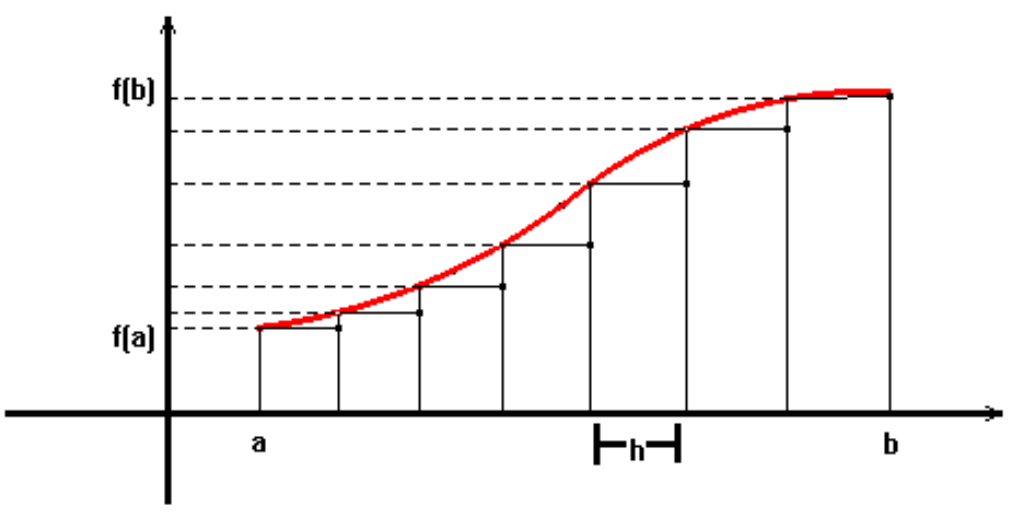

Figura 1. Método dos Retângulos

Sendo $\boldsymbol{n}$ o número de partições estabelecido no intervalo $[\boldsymbol{a}, \boldsymbol{b}]$, define-se $\boldsymbol{h}$ como:

$$
h=\frac{b-a}{n}
$$

Observando a Figura 1, tem-se $\boldsymbol{n}=\mathbf{7}$. A partir daí, determinam-se as áreas dos retângulos.

Sendo a área total sob a curva:

$$
\begin{aligned}
& \text { Area Total }=A_{0}+A_{1}+A_{2}+A_{9}+A_{4}+A_{6}+A_{6}+A_{7} \\
& =h\left[f\left(x_{0}\right)+f\left(x_{1}\right)+f\left(x_{2}\right)+f\left(x_{9}\right)+f\left(x_{4}\right)+f\left(x_{6}\right)+f\left(x_{6}\right)+f\left(x_{7}\right)\right]
\end{aligned}
$$


INTNUM: Uma Ferramenta Computacional para Visualização e Cálculo dos Métodos de Integração Numérica

Bruno Nunes Myrrha Ribeiro - Carlos Vitor de Alencar Carvalho

Generalizado, do caso acima, obtém-se a área total no Método dos Retângulos:

$$
\begin{gathered}
\text { Area Total }=h\left[f\left(x_{0}\right)+f\left(x_{1}\right)+f\left(x_{2}\right)+\cdots+f\left(x_{n-2}\right)+f\left(x_{n-1}\right)+f\left(x_{n}\right)\right] \\
=\Sigma_{t=0}^{n} h f\left(x_{i}\right) \\
\simeq \int_{x_{0}}^{x_{n}} f(x) d x
\end{gathered}
$$

\subsection{Método dos Trapézios}

Consiste na aproximação numérica, por trapézios, em relação à área sob a função dada.

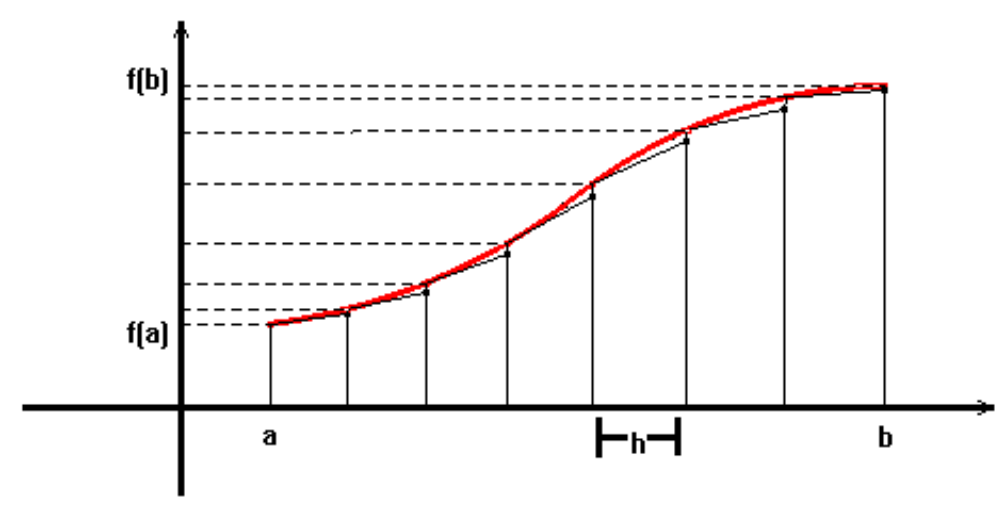

Figura 2. Método dos Trapézios.

Sendo $\boldsymbol{n}$ o número de partições estabelecido no intervalo $[\boldsymbol{a}, \boldsymbol{b}]$, define-se $\boldsymbol{h}$ como:

$$
h=\frac{b-a}{n}
$$

Observada a Figura 2, tem-se $\boldsymbol{n}=\mathbf{7}$. A partir daí, determinam-se as áreas dos trapézios.

Sendo a área total sob a curva:

$$
\begin{gathered}
\text { Areat } \operatorname{Total}=A_{0}+A_{1}+A_{2}+A_{g}+A_{4}+A_{6}+A_{6} \\
=\frac{h}{2}\left[f\left(x_{0}\right)+2 f\left(x_{1}\right)+2 f\left(x_{2}\right)+2 f\left(x_{1}\right)+2 f\left(x_{4}\right)+2 f\left(x_{1}\right)+2 f\left(x_{4}\right)+f\left(x_{7}\right)\right]
\end{gathered}
$$

Generalizado, do caso acima, obtém-se a área total no Método dos Trapézios: 
INTNUM: Uma Ferramenta Computacional para Visualização e Cálculo dos Métodos de Integração Numérica

Bruno Nunes Myrrha Ribeiro - Carlos Vitor de Alencar Carvalho

$$
\begin{gathered}
\text { Area Total }=\frac{h}{2}\left[f\left(x_{0}\right)+2 f\left(x_{1}\right)+2 f\left(x_{2}\right)+\cdots+2 f\left(x_{n-2}\right)+2 f\left(x_{n-1}\right)+f\left(x_{n}\right)\right] \\
=\frac{h}{2}\left[f(x)+\sum_{t=1}^{n-1} 2 f\left(x_{t}\right)+f(b)\right] \\
\propto \int_{x_{0}}^{x_{n}} f(x) d x
\end{gathered}
$$

\subsection{Método de Simpson}

Consiste na aproximação numérica, por trapézios parabólicos, em relação à área sob a função dada.

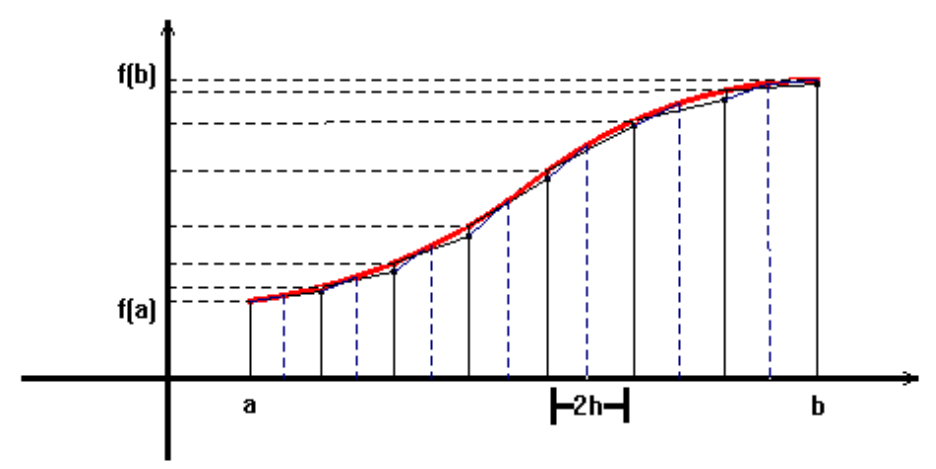

Figura 3. Método de Simpson.

Sendo $\boldsymbol{n}$ o número de partições, com $\boldsymbol{n}$ par, estabelecido no intervalo $[\boldsymbol{a}, \boldsymbol{b}$ ], define-se $h$ como:

$$
h=\frac{b-a}{2 n}
$$

Apresenta-se cada área como um trapézio parabólico, tomada com uma interpolação quadrática na limitação $\mathbf{2} \boldsymbol{h}$ em três pontos $\boldsymbol{x}_{0}, \boldsymbol{x}_{\mathbf{1}}$ e $\boldsymbol{x}_{\mathbf{2}}$ (Cuminato, 2009), logo:

$\left.A_{0}=\int_{y_{0}}^{x_{1}} f(x) d x \stackrel{ }{=} \frac{h}{3}\left[f\left(x_{0}\right)+4 f\left(x_{1}\right)+f\left(x_{2}\right)\right]=\frac{h}{3}\left[f(a)+4 f\left(x_{1}\right)+f\left(x_{2}\right)\right)\right]$

De forma análoga aos métodos descritos anteriormente, sendo a área total sob a curva: 
INTNUM: Uma Ferramenta Computacional para Visualização e Cálculo dos Métodos de Integração Numérica

Bruno Nunes Myrrha Ribeiro - Carlos Vitor de Alencar Carvalho

$$
\begin{aligned}
\text { Area Total }=A_{0}+ & A_{1}+A_{2}+A_{9}+A_{4}+A_{6}+A_{6} \\
& =\frac{h}{3}\left[f\left(x_{0}\right)+4 f\left(x_{1}\right)+2 f\left(x_{p}\right)+f\left(x_{14}\right)\right]
\end{aligned}
$$

Com:

$$
\begin{gathered}
f\left(x_{1}\right)=f\left(x_{1}\right)+f\left(x_{1}\right)+f\left(x_{1}\right)+f\left(x_{7}\right)+f\left(x_{9}\right)+f\left(x_{11}\right)+f\left(x_{18}\right) \\
f\left(x_{7}\right)=f\left(x_{2}\right)+f\left(x_{4}\right)+f\left(x_{6}\right)+f\left(x_{8}\right)+f\left(x_{10}\right)+f\left(x_{12}\right)
\end{gathered}
$$

Generalizado, do caso acima, obtém-se a área total no Método de Simpson:

$$
\begin{aligned}
& \text { Area Total }= \\
& \begin{aligned}
& \frac{h}{3}\left[f\left(x_{0}\right)+4\left(f\left(x_{1}\right)+\cdots+f\left(x_{2 n-1}\right)\right)+2\left(f\left(x_{2}\right)+\cdots+f\left(x_{2 n-2}\right)\right)\right. \\
& \left.+f\left(x_{2 n}\right)\right] \\
=\frac{h}{d}[f(a) & \left.+\sum_{i=0}^{2 n-1} 4 f\left(x_{2 t+1}\right)+\sum_{t=0}^{2 n-2} 2 f\left(x_{2 t+2}\right)+f(b)\right] \\
& \cong \int_{x_{0}}^{x_{2 n}} f(x) d x
\end{aligned}
\end{aligned}
$$

\section{Desenvolvimento Computacional}

O software foi desenvolvido em linguagem C++, no ambiente Microsoft Visual C++ 2008 Express Edition, para o qual usou-se o sistema gráfico OpenGL (Woo et al. 1999). Nesta seção são apresentados os algoritmos dos métodos implementados.

Basicamente, pode-se dividir o desenvolvimento em três algoritmos principais: algoritmo 1 - valor da integral, algoritmo 2 - visualização da função, algoritmo 3 - visualização do método de integração.

\subsection{Algoritmo 1 - Valor da Integral}

Destina-se à aproximação numérica do cálculo da integral, mediante os métodos citados anteriormente, o que define o número de partições. O quadro 1 mostra detalhes do algoritmo. 
INTNUM: Uma Ferramenta Computacional para Visualização e Cálculo dos Métodos de Integração Numérica

Bruno Nunes Myrrha Ribeiro - Carlos Vitor de Alencar Carvalho

Quadro 1: Algoritmo 1 - Valor da Integral.

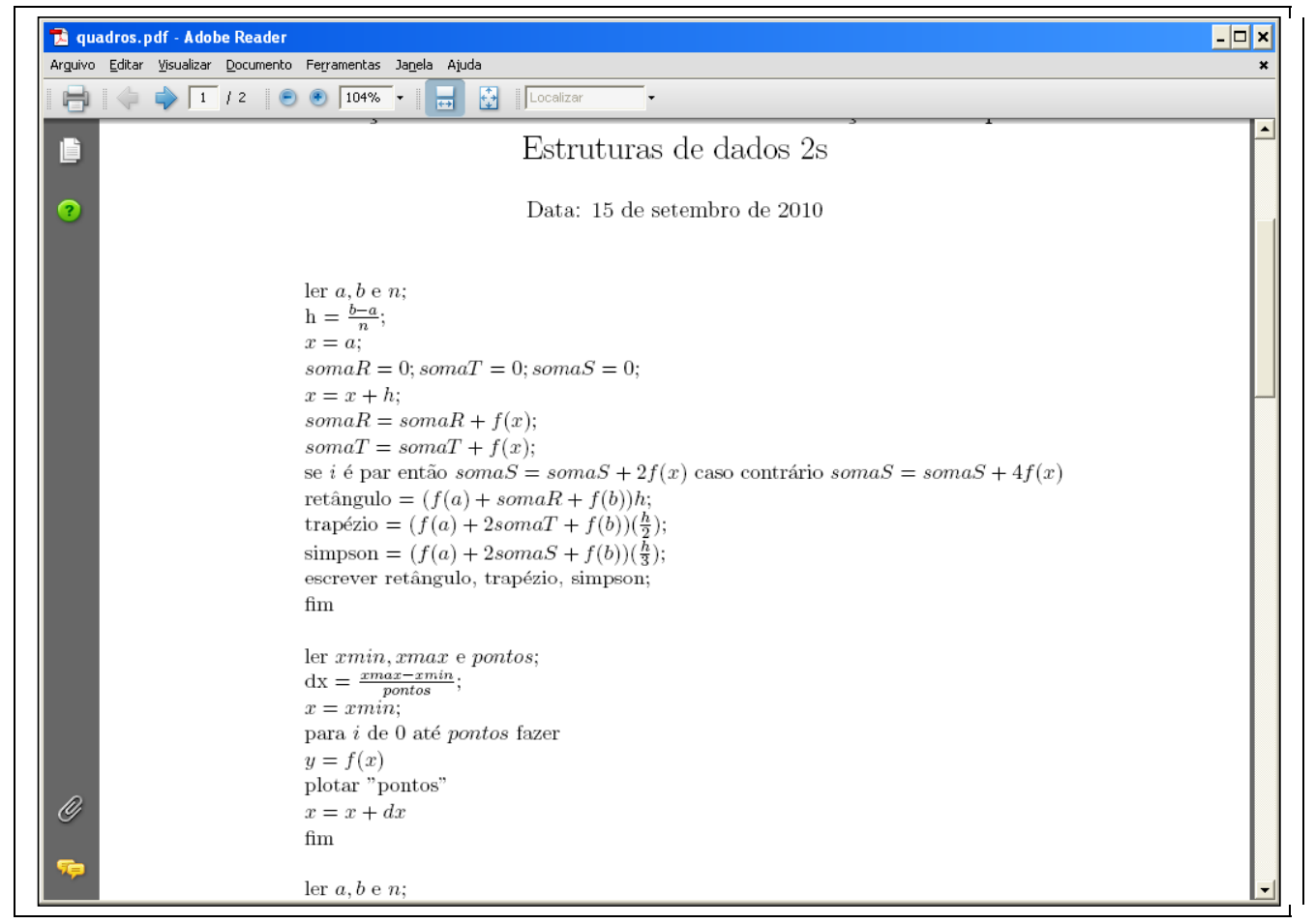

\subsection{Algoritmo 2 - Visualização da Função}

Cria o gráfico da função dada, ao utilizar visualização por pontos. O quadro 2 mostra detalhes do algoritmo.

Quadro 2 - Algoritmo 2: Visualização da Função.

\begin{tabular}{|l|}
\hline ler $x$ min, xmax e pontos; \\
$\mathrm{dx}=\frac{x \max -x \min }{\text { pontos }} ;$ \\
$x=x$ min; \\
para $i$ de 0 até pontos fazer \\
$y=f(x)$ \\
plotar "pontos" \\
$x=x+d x$ \\
fim
\end{tabular}


INTNUM: Uma Ferramenta Computacional para Visualização e Cálculo dos Métodos de Integração Numérica

Bruno Nunes Myrrha Ribeiro - Carlos Vitor de Alencar Carvalho

\subsection{Algoritmo 3 - Visualizar Métodos de Integração}

Estabelece parâmetros para cada um dos métodos, e busca a visualização por meio de lista de quadriláteros. O quadro 3 mostra detalhes do algoritmo.

\section{Quadro 3: Algoritmo 3 - Visualização dos Métodos de Integração.}

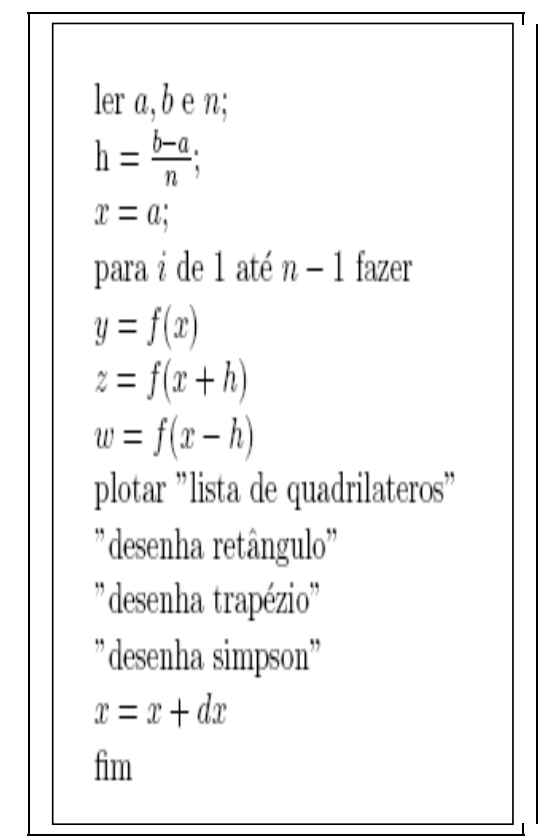

\section{Resultados}

Na Figura 4 é apresentado o "menu” de opções do programa. Nele, o usuário pode inserir primeiramente, como dado de entrada, a escolha entre os métodos de integração numérica: Ponto à Esquerda, Trapézio e Simpson. Após o método ser selecionado, insere-se o número correspondente à quantidade de partições iniciais, o que possibilita uma interação, pelo teclado (com as teclas “+” e “-”), com a finalidade de variação entre as partições, o que resulta na visualização e no valor da integral. Num primeiro momento seleciona o método e a quantidade inicial de partições " $n$ ". 
INTNUM: Uma Ferramenta Computacional para Visualização e Cálculo dos Métodos de Integração Numérica

Bruno Nunes Myrrha Ribeiro - Carlos Vitor de Alencar Carvalho

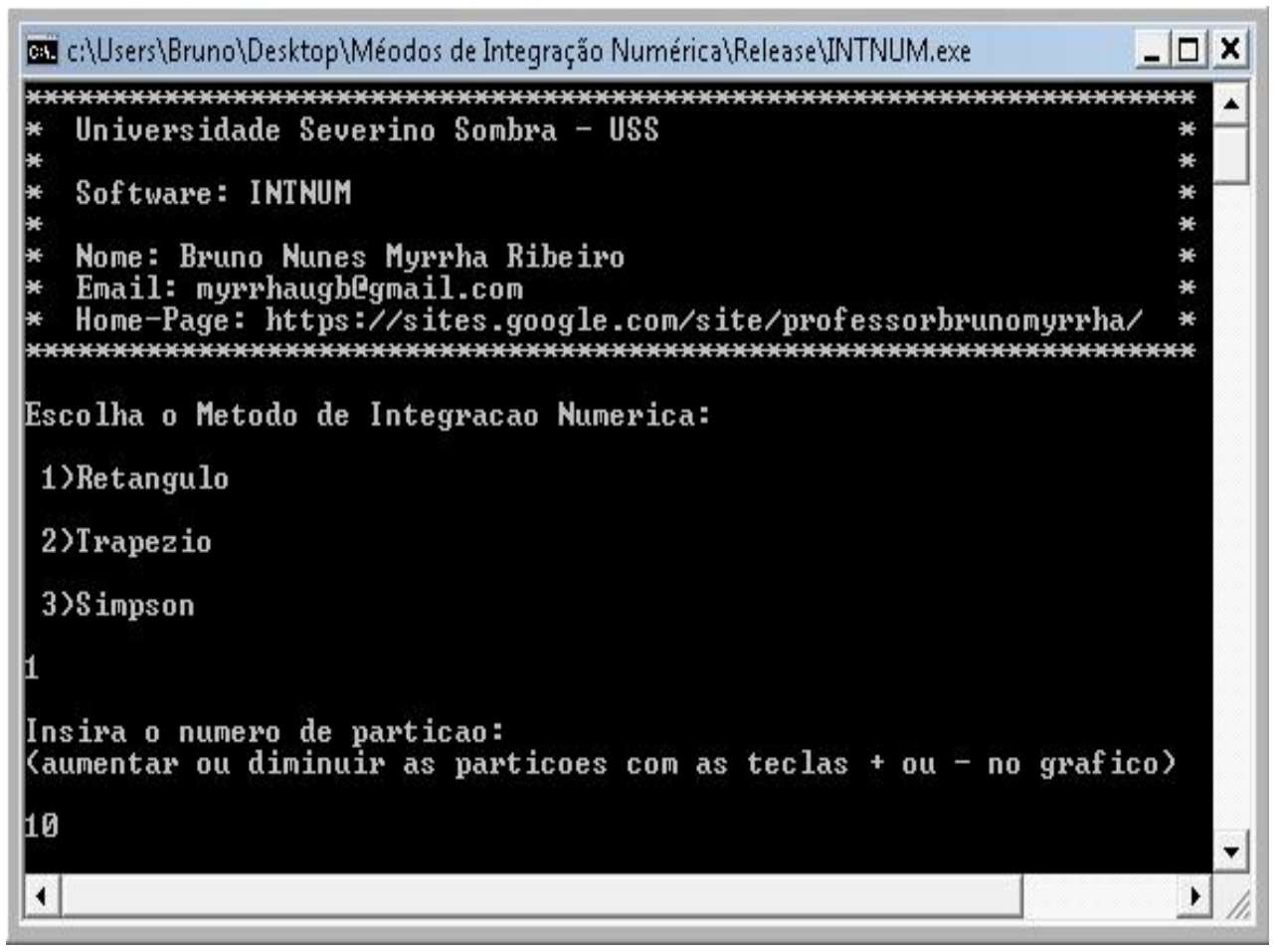

Figura 4. Menu.

\subsection{Método dos Retângulos}

Para o método dos Retângulos foram utilizadas a função e as condições descritas na tabela 1 .

Tabela 1: Condições iniciais para testar o método dos retângulos.

\begin{tabular}{|c|c|c|c|c|}
\hline Função & $\begin{array}{c}\text { Número de } \\
\text { partição }\end{array}$ & $\begin{array}{c}\text { Variação do } \\
\text { domínio }\end{array}$ & $\begin{array}{c}\text { Intervalo de } \\
\text { integração }\end{array}$ & $\begin{array}{c}\text { Valor da } \\
\text { integral }\end{array}$ \\
\hline $\boldsymbol{f}(\boldsymbol{x})=\boldsymbol{x} \operatorname{sen} \boldsymbol{x} \cos \boldsymbol{x}$ & 25 & {$[-10,10]$} & {$[-10,10]$} & 15,5223 \\
\hline
\end{tabular}


INTNUM: Uma Ferramenta Computacional para Visualização e Cálculo dos Métodos de Integração Numérica

Bruno Nunes Myrrha Ribeiro - Carlos Vitor de Alencar Carvalho

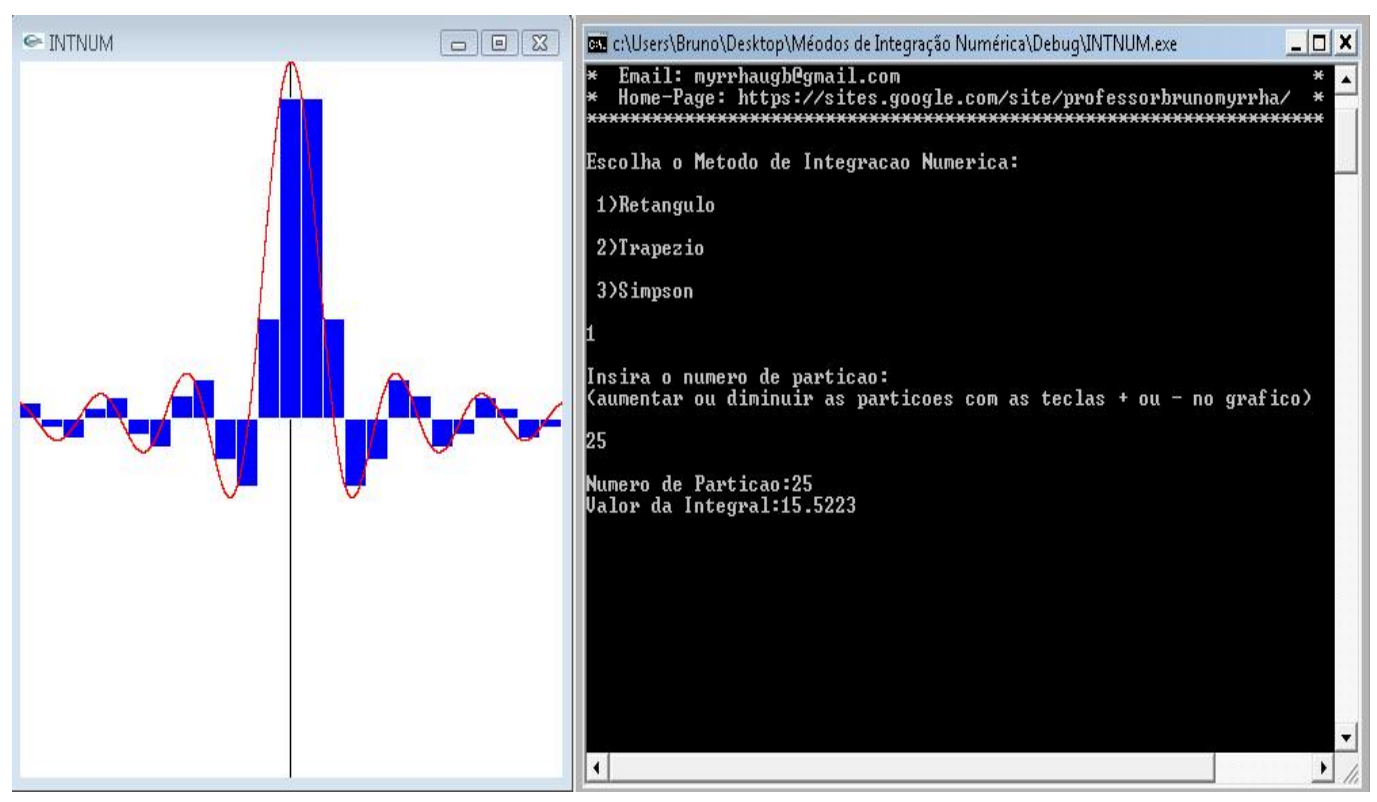

Figura 5. Método dos Retângulos (25 partições).

Na Figura 6, aumenta o número de partição para 50 ao se utilizar a interação via teclado.

Tabela 2: Aumento de partições para testar o método dos retângulos.

\begin{tabular}{|c|c|c|c|c|}
\hline Função & $\begin{array}{c}\text { Número de } \\
\text { partição }\end{array}$ & $\begin{array}{c}\text { Variação do } \\
\text { domínio }\end{array}$ & $\begin{array}{c}\text { Intervalo de } \\
\text { integração }\end{array}$ & $\begin{array}{c}\text { Valor da } \\
\text { integral }\end{array}$ \\
\hline $\boldsymbol{f}(\boldsymbol{x})=\boldsymbol{x} \operatorname{sen} \boldsymbol{x} \cos \boldsymbol{x}$ & 50 & {$[-10,10]$} & {$[-10,10]$} & 15,4922 \\
\hline
\end{tabular}

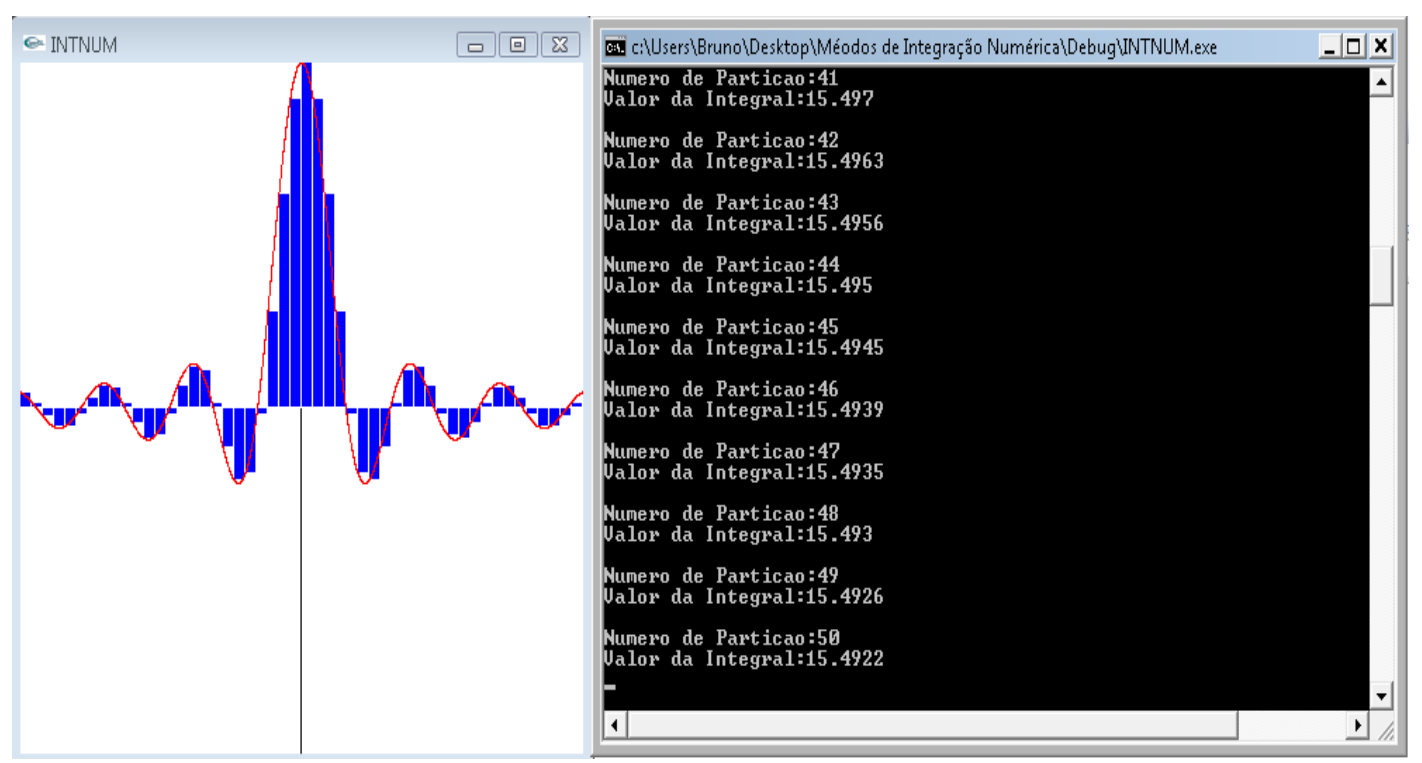

Figura 6. Método dos Retângulos (25 partições)

Revista Eletrônica TECCEN, Vassouras, v. 3, n. 2, Edição Especial, p. 71-84, abr./jun., 2010 
INTNUM: Uma Ferramenta Computacional para Visualização e Cálculo dos Métodos de Integração Numérica

Bruno Nunes Myrrha Ribeiro - Carlos Vitor de Alencar Carvalho

\subsection{Método dos Trapézios}

Tabela 3: Condições iniciais para testar o método dos trapézios.

\begin{tabular}{|c|c|c|c|c|}
\hline Função & $\begin{array}{c}\text { Número de } \\
\text { partição }\end{array}$ & $\begin{array}{c}\text { Variação do } \\
\text { domínio }\end{array}$ & $\begin{array}{c}\text { Intervalo de } \\
\text { integração }\end{array}$ & $\begin{array}{c}\text { Valor da } \\
\text { integral }\end{array}$ \\
\hline $\boldsymbol{f}(\boldsymbol{x})=\boldsymbol{x} \operatorname{sen} \boldsymbol{x} \cos \boldsymbol{x}$ & 50 & {$[-10,10]$} & {$[-10,10]$} & 15,4922 \\
\hline
\end{tabular}

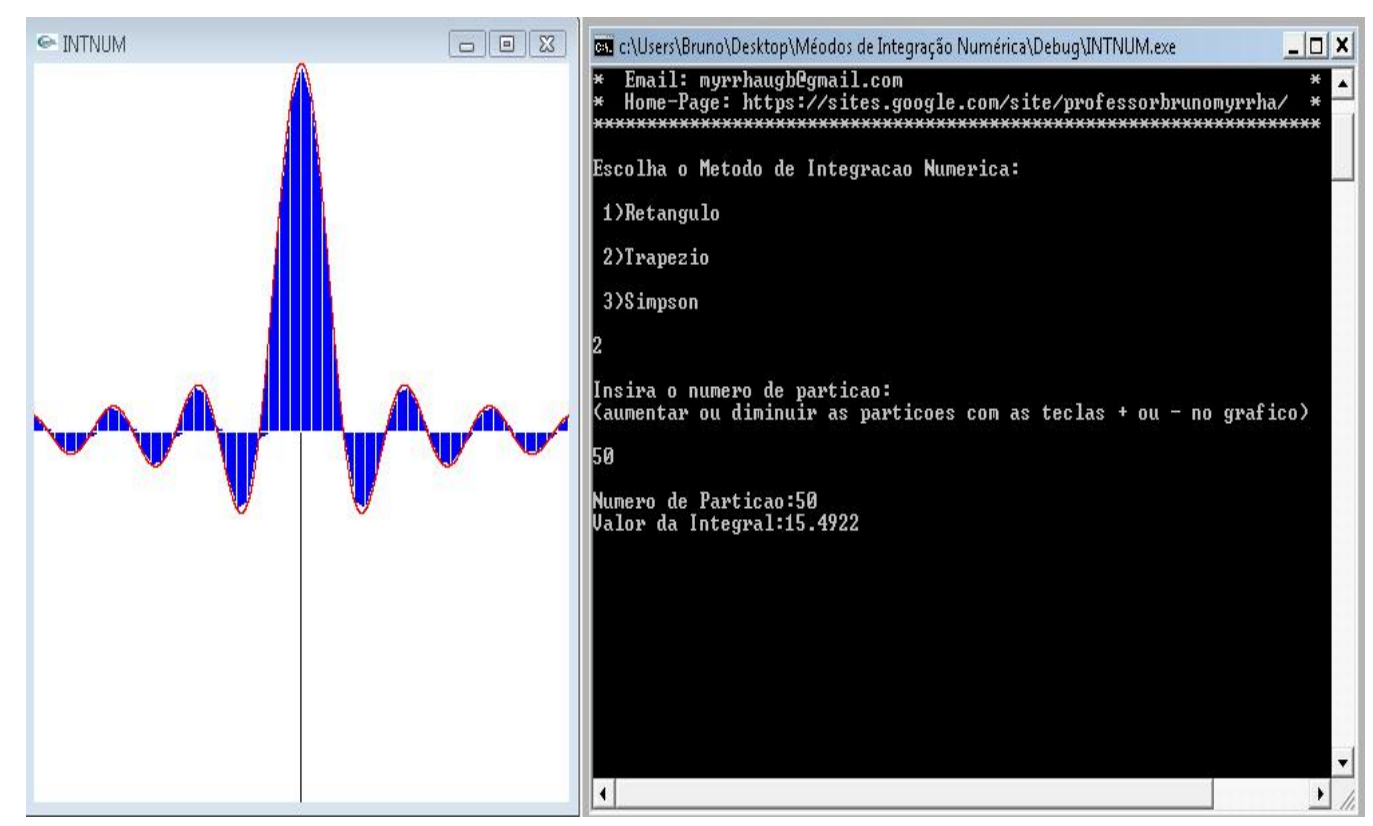

Figura 7. Método dos Trapézios (50 partições).

\subsection{Método de Simpson}

Tabela 4: Condições iniciais para testar o método de Simpson.

\begin{tabular}{|c|c|c|c|c|}
\hline Função & $\begin{array}{c}\text { Numero de } \\
\text { partição }\end{array}$ & $\begin{array}{c}\text { Variação do } \\
\text { domínio }\end{array}$ & $\begin{array}{c}\text { Intervalo de } \\
\text { integração }\end{array}$ & $\begin{array}{c}\text { Valor da } \\
\text { integral }\end{array}$ \\
\hline $\boldsymbol{f}(\boldsymbol{x})=\boldsymbol{x} \operatorname{sen} \boldsymbol{x} \cos \boldsymbol{x}$ & 50 & {$[-10,10]$} & {$[-10,10]$} & 15,4821 \\
\hline
\end{tabular}


INTNUM: Uma Ferramenta Computacional para Visualização e Cálculo dos Métodos de Integração Numérica

Bruno Nunes Myrrha Ribeiro - Carlos Vitor de Alencar Carvalho

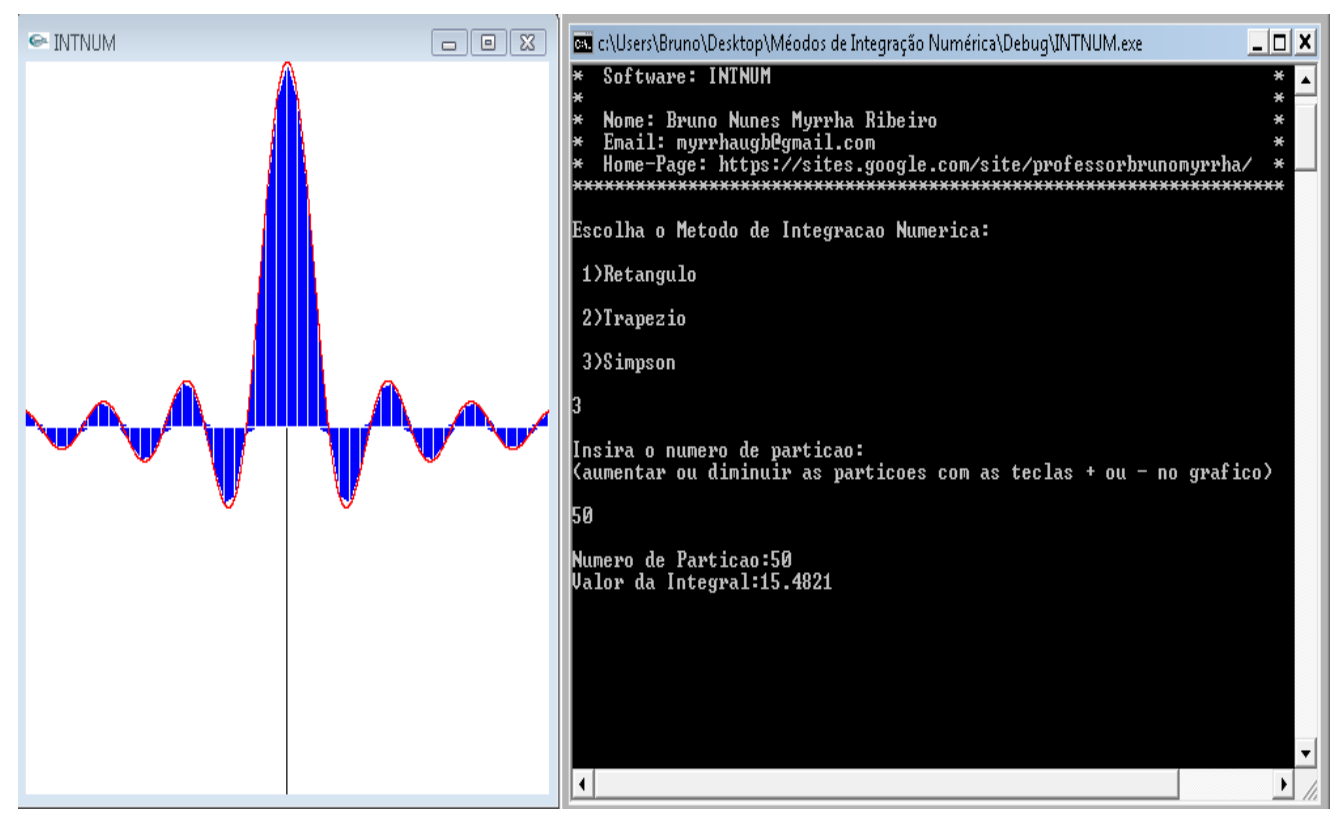

Figura 8. Método de Simpson (50 partições).

\section{Considerações Finais}

Este artigo se refere ao desenvolvimento do software INTNUM. A possibilidade que o software tem de permitir a visualização dos métodos de integração numérica, bem como os respectivos valores da integral e a interatividade por meio do teclado em relação à aproximação da área sob a curva, aumenta significativamente a assimilação mais sólida em relação ao conteúdo "Integral Numérica”, na disciplina de Cálculo Numérico, e proporciona uma aula mais dinâmica.

Entretanto, é importante que os educadores sejam mediadores e direcionadores do aprendizado e mostrem que os recursos do software desenvolvido fornecerão respostas de acordo com os dados que o aluno irá colocar, o que leva a análises sobre os resultados obtidos. A próxima etapa desse trabalho será em torno do desenvolvimento de uma interface gráfica mais amigável e a utilização do software com alunos que cursam a disciplina de Cálculo Numérico para que se verifiquem sua avaliação/validação em sala de aula. 
INTNUM: Uma Ferramenta Computacional para Visualização e Cálculo dos Métodos de Integração Numérica

Bruno Nunes Myrrha Ribeiro - Carlos Vitor de Alencar Carvalho

\section{Referências}

Borba, M. C., Penteado, M. G.. (2003) Informática e Educação Matemática. 3.ed. Belo Horizonte: Autêntica.

Cuminato, J. A., Cálculo Numérico, Apostila do ICMC/USP. (texto auxiliar).

Castilho, J. E. (2001)

D’Ambrósio, Ubiratan. (2002) Educação Matemática - da teoria à prática. 9a ed. São Paulo: Papirus, Coleção Perspectivas em Educação Matemática.

Foley, J.D., Van Dam, A., Feiner, S. K. and Heghes, J. F. (1999) Computer Graphics Principles and Practice, second edition in C, Addison-Wesley publish company.

Paiva, A.M.S., Carvalho, J.V., Carvalho, C.V.A., Porto, I.P.G., (2008) “A integração da TIC na Escola Básica: Questões para Avaliação”, Revista Eletrônica TECCEN, ano 1, vol 1.

Pereira, T. P. (2008) “Cálculo numérico computacional”. Sobral: UVA.

Valente, J. A. (2003) Diferentes Usos do Computador na Educação. Em J.A. Valente (Org.), Computadores e Conhecimento: repensando a educação (pp.1-23). Campinas, SP: Gráfica da UNICAMP.

Tajra, S. F. (2003) Informática na educação: novas ferramentas pedagógicas para o professor da atualidade. 3. ed. São Paulo: Érica.

Woo, M., Jackie, N, Davis, J., Shreiner, D. (1999) OpenGL Programming Guide, Third Edition (OpenGL, Version 1.2), Addison-Welsey.

Carvalho, C. V. A.; Carvalho, J. V.; Aguiar, R. A. (2009) Utilização do software livre OCTAVE para apoio ao ensino do Cálculo Numérico. In: VI CIBEM-Congresso Iberoamericano de Educación Matemática, Poerto Montt, vol. 1. p. 679-684.

Carvalho, C. V. A, Martha, L. F.; Teixeira, (2005) W. FLUXOVENTO - Um simulador gráfico interativo para o estudo de ventilação em ambientes construídos. In: VII Encontro Nacional sobre Conforto no Ambiente Construído (ENCAC). ISBN: 85-89478-12-2. Maceió, v. 1. p. 350-360.

Haidt, R. C. C. (2001) Curso de didática Geral, São Paulo - Brasil: Editora Ática - Série Educação. 